\title{
Characterizing the Geometry and Footprint of the DELVE Survey
}

Tom McDonald - Waubonsee Community College

Javier Sanchez - Fermilab

FERMILAB-POSTER-21-097-STUDENT

\section{What is DELVE?}

The DECam Local Volume Exploration Survey (DELVE) aims to study the faint and distant galaxies which are heavily dominated by dark matter. By studying these galaxies, DELVE will be instrumental in further understanding dark matter.

\section{What I Worked On}

My main job was to work on the survey geometry characterization of DELVE, specifically to use maps to see where we have data, and where we don't. This was done through use of a program called 'Decasu', which generated accurate maps based on the data collected.

\section{Moving Forward}

We still wonder, what will be the best observing conditions for accurate galaxy count. What conditions will be best for a final catalogue? Once we can determine these patterns in observing conditions, we will be able to decrease their overall influence in order to build the most accurate catalogue possible.

\section{Project Goals}

As a broad goal, we want to observe the angular distribution of galaxies in the sky in order to obtain information about dark energy and dark matter.

\section{Survey Geometry Goals}

We know that actual number of galaxies in a given part of the sky does not depend on the nightly observing conditions, so we must be carefully and accurately measured to avoid any confusion. We'll need as much information as possible about nightly observing conditions in order to follow their patterns and reduce their contamination with data taken regarding actual galaxies.

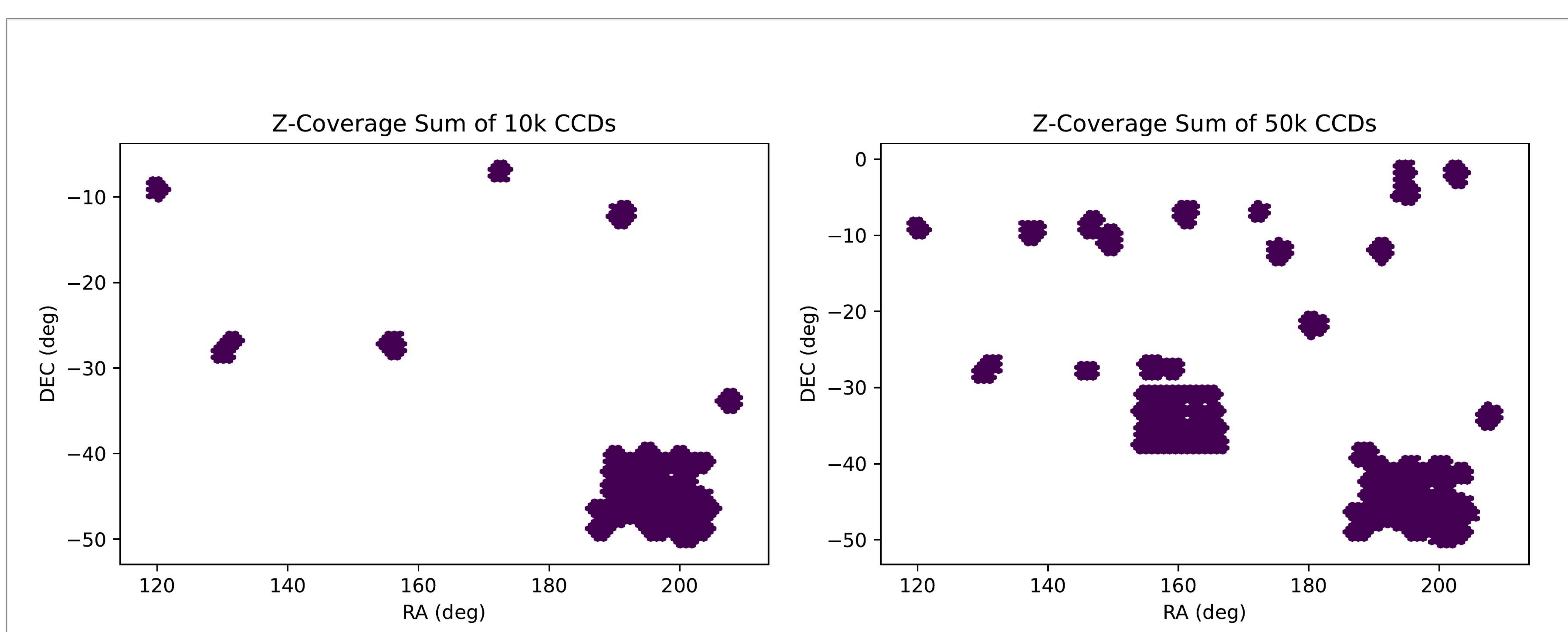

A sample of the survey geometry maps. In the figure, we represent the coverage of the footprint.

\section{Special Acknowledgements}

I would like to personally thank Dr. Javier Sanchez, Dr. Alex DrlicaWagner, and the rest of the DELVE team for all their encouragement, patience, and support this summer. I had an incredible time and feel grateful to have had this opportunity. Thank you for having me!

This manuscript has been authored by Fermi Research Alliance, LLC under Contract No. DE-AC02 $07 \mathrm{CH} 11359$ with the U.S. Department of Energy, Office of Science, Office of High Energy Physics. 